\title{
EFIS Lecture: Understanding the CTLA-4 checkpoint in the maintenance of immune homeostasis
}

\author{
Lucy S. K. Walker
}

Institute of Immunity \& Transplantation, University College London Division of Infection \& Immunity, Royal Free Campus, London, UK NW3 2PF

Corresponding author: Prof Lucy S.K. Walker, Institute of Immunity \& Transplantation, University College London Division of Infection \& Immunity, Royal Free Campus, London, UK NW3 2PF. Tel: +44 (0)20 77940500 ext 22468. Email: lucy.walker@ucl.ac.uk

keywords: CTLA-4, autoimmunity, T-cells, costimulation, CD28, tolerance 


\section{Abstract}

The past 20 years have heralded fascinating developments in the field of CTLA-4 biology. The CTLA-4 protein is a critical negative regulator of T cell immunity and its absence provokes severe lymphoproliferative disease. In a surprising twist, the generation of mixed bone marrow chimeric mice revealed that CTLA-4 predominantly functions in a cell-extrinsic manner, suggesting that CTLA-4 expressed on one cell can modify the behaviour of another cell. This was followed by the demonstration that CTLA-4 is highly expressed in regulatory T cells and can contribute to their suppressive activity. In line with a cell-extrinsic function, increasing evidence indicates that CTLA-4positive cells can modify the phenotype of antigen presenting cells (APC), thereby regulating the priming of naive T cells. Notably, CTLA-4 is able to downregulate expression of costimulatory ligands on APC via a process of trans-endocytosis. The identification of patients with mutations in the ctla4 gene has provided an opportunity to study the contribution of CTLA-4 to Treg function and immune regulation in the human immune system. Finally, it has become apparent that CTLA-4 also plays a role in controlling humoral immunity, via the regulation of CD28-driven follicular helper T cell differentiation. At the recent German Society for Immunology congress, I discussed some of the contributions of my own lab to the unfolding of the CTLA-4 story, in the context of the work of others in the field. Despite the enormous clinical potential associated with modulation of the CTLA-4 pathway, including the use of soluble CTLA-4 molecules in autoimmune settings and blocking antibodies in cancer, it is clear there is still much to learn about this important pathway. 


\section{Introduction}

Understanding CTLA-4-based immunoregulation poses an unusual conceptual challenge. We are faced with a pair of homologous receptors expressed on T cells, CTLA-4 and CD28, that share the same ligands yet have completely opposing biological functions[1](Figure 1). CD28 is an important costimulatory molecule providing the critical "second signal" that permits full T cell activation; genetic deficiency of $c d 28$ therefore results in markedly impaired $\mathrm{T}$ cell immunity [2,3]. Conversely, key experiments in the mid 1990s established that ablation of the ctla4 gene unleashes aggressive immune hyperactivation with multi-organ lymphocytic infiltration and early death $[4,5]$. Thus, the biological functions directed by CD28 and CTLA-4 could not be more different. The notion that these two receptors share the same ligands, despite their polar opposite functions, is therefore somewhat counterintuitive. How can the same ligands safely direct both immunity and tolerance?

The complexities of the system do not stop there. The striking phenotype of ctla4 knockout mice highlights the importance of CTLA-4 inhibitory function, yet despite binding to cell surface ligands CTLA-4 appears surprisingly reluctant to present itself at the cell surface, instead spending the majority of its time in intracellular vesicles. Indeed, any CTLA-4 molecules reaching the cell membrane internalise again within minutes. This is no accident - the constitutive endocytosis of CTLA-4 is hardwired via an amino acid sequence in its cytoplasmic tail, which contains a motif that couples it to the clathrin adaptor complex AP-2[6-8]. The entire cytoplasmic sequence of CTLA-4 is highly conserved evolutionarily with virtually no deviations being tolerated in mammals - your own CTLA-4 cytoplasmic sequence is $100 \%$ identical to that found in mice and in 
dolphins. So, if you're wondering what you've got in common with Donald Trump (and Donald Duck?) - it's probably your CTLA-4.

Why would such a critical immune regulator be so coy in making itself available for ligand engagement? How can the same ligands safely trigger both immunity via CD28 and regulation via CTLA-4? Clearly the CTLA-4 system represents a departure from normal immunological paradigms and new models must be invoked to fully understand it.

\section{CTLA-4 functions in a cell extrinsic manner}

The late 1990's was an exciting time for CTLA-4 research. We had embraced the idea of a "two-signal model" of T cell activation, whereby triggering the TCR in the absence of CD28 led to antigen-specific unresponsiveness (clonal anergy) $[9,10]$ - suddenly it looked as though CTLA-4 might add an extra dimension to that model. Perhaps functional unresponsive was not a passive affair, arising from the absence of costimulation, but instead was being actively reinforced by an inhibitory receptor, namely CTLA-4[11-13]. It looked increasingly as though the balance between CD28 and CTLA-4 signaling was instrumental in dictating whether a T cell encounter with antigen was tolerogenic or immunogenic. Indeed, it was clear that the CD28 and CTLA-4 pathways had completely opposite effects on the functional responses of T cells in vitro[14-18]. Mechanistic data suggested that CTLA-4 might transmit signals via the tyrosine phosphatase SHP-2[19, 20] leading to inhibition of TCR-mediated cellular 
activation by targeting the RAS pathway[19] or promoting TCR-zeta

dephosphorylation[20].

Just as things seemed to be falling into place, the field was rocked by an extraordinary finding. Despite the fact that CTLA-4-deficient mice exhibited a lethal lymphoproliferative syndrome, bone marrow chimeras containing a mixture of wildtype and CTLA-4-deficient cells appeared to be completely normal. Not only were they healthy, but the CTLA-4-deficient T cells in them had a normal resting phenotype rather than a hyperactivated one[21](Figure 2). This was hard to reconcile with the idea that CTLA-4 worked by transmitting inhibitory signals into the T cell expressing it - in that case, the T cells bearing CTLA-4 would be appropriately regulated but those lacking it would not. This simple chimera experiment has since become something of an "elephant in the room" in the CTLA-4 field. Numerous mechanisms have been proposed whereby CTLA-4 controls the activation, adhesion or motility of the T cells expressing it. Yet the sequelae of these arguments is that CTLA-4-expressing cells in mixed bone marrow chimeras would be expected to exhibit altered activation, adhesion or motility, compared with the T cells lacking CTLA-4. Why then is there no evidence of this? The bone marrow chimera experiment has been widely reproduced by multiple labs[22-27] and in all cases CTLA-4+/+ cells efficiently prevent hyperactivation of CTLA-4-/- cells. The chimeric mice have also been subjected to a range of parasitic[23] or viral[22, 23] infections so that the biological response of the T cells to immunological challenge could be examined. In arguably the most comprehensive of these studies, antigen-specific CD4 and CD8 T cell responses to 8 different viral epitopes were tracked. Antigen-driven proliferation, acquisition of effector function, T cell repertoire selection, functional avidities, memory formation and response to re-challenge were found to be essentially 
indistinguishable between CTLA-4-deficient and CTLA-4-sufficient T cells in chimeric mice[22]. Thus, there appears to be no detectable cell-intrinsic consequence of expressing the CTLA-4 protein. Putative molecular mechanisms for CTLA-4 function must therefore take into account the strong evidence that CTLA-4 operates in a cellextrinsic manner in vivo.

\section{Role of CTLA-4 in Treg function}

A cell-extrinsic function for CTLA- 4 implies that cells expressing CTLA-4 are able to regulate the behaviour of cells lacking CTLA-4. One obvious paradigm of cell-extrinsic regulation is the control of the immune system by regulatory T cells (Treg). This specialised subset of CD4 T cells acts in a dominant suppressive manner to inhibit the induction of autoimmune diseases[28, 29]. Mice lacking Treg, due to a defect in the transcription factor Foxp3, develop a lethal lymphoproliferative disorder highly reminiscent of that seen in CTLA-4 deficiency[30-32] and humans with mutations in Foxp3 also exhibit an aggressive immune dysregulation syndrome[33, 34]. It therefore caused great excitement when it was shown that Treg are the major cell population expressing CTLA-4[35, 36]. CTLA-4 is constitutively expressed intracellularly at high levels in the Treg population but is lacking from naive conventional T cells and is only induced de novo following cellular activation. These observations prompted intense speculation over whether CTLA-4 is responsible for mediating the suppressive function of Treg. However, testing this idea experimentally proved surprisingly difficult. Attempts to block the function of Treg with CTLA-4 antibodies in vitro produced mixed results, with some studies demonstrating reduced Treg suppression[36, 37] and some 
not[29]. In one study, different preparations of the same antibody clone yielded differing results[38]. When Treg from CTLA-4-deficient mice were used, these generally exhibited suppressive function in vitro, sometimes as efficiently as wildtype Treg[37, 39, 40] and sometimes slightly less so[36]. A major confounder for many of these studies was that the CTLA-4-deficient Treg were being isolated from mice that were in the process of developing lymphoproliferative disease. Since disease in CTLA-4-deficient mice results from unimpinged engagement of the CD28 pathway, it can be abrogated by blocking CD28 signaling[41-43], theoretically removing this confounder. However the known requirement of CD28 for normal Treg generation and homeostasis[44, 45] poses a limitation here: to specifically test to role of CTLA-4 in Treg, ideally one would want to interrupt CTLA-4 pathway whilst leaving the CD28 pathway intact.

Around the time that these issues were being debated, my group was studying double transgenic mice expressing ovalbumin (OVA) in the pancreas under the rat insulin promoter and also expressing the OVA-specific TCR transgene, D011.10. This model was not originally intended to be used for Treg analysis but rather was designed to study the early events in type 1 diabetes pathogenesis. However, as is often the case in science, unexpected findings can open the door to new insights. Realising that the rat insulin promoter was active in the thymus, and that by driving expression of OVA at this site it was triggering Treg differentiation[46], we began to consider the potential advantages of having a TCR transgenic model of Treg differentiation. Most notable among these was the idea that by breeding these animals to a CTLA-4 deficient strain we would be able to generate antigen-specific Treg that lacked CTLA-4 but retained an intact CD28 pathway. To avoid any variation in the antigen specificity of the Treg, we bred the mice to a rag deficient background (precluding expression of endogenous TCR- 
$\alpha$ chains). This had the added advantage of avoiding any residual off-target activation of the CTLA-4 deficient T cells, since now their specificity was strictly restricted to the ovalbumin expressed in the pancreatic islets.

For the first time we were able to test the suppressive function of CTLA-4-deficient Treg bearing an identical specificity and affinity for antigen as their wildtype counterparts (both being D011.10+rag-/-), that had an intact CD28 pathway, and that had not been isolated from mice with lymphoproliferative disease. By purifying OVA-specific Treg from CTLA-4-sufficient or CTLA-4-deficient mice we were able to demonstrate that CTLA-4 expression was absolutely required for regulation of the autoimmune response against pancreatic islets in vivo[40] (Figure 3). This contrasted with the results obtained in vitro using the same Treg populations[40], suggesting that in vitro suppression assays do not always faithfully recapitulate in vivo scenarios. A similar message came from the work of Sojka et al[39] who found that CTLA-4 deficient Treg were incapable of suppressing lymphopenia-induced T cell expansion in vivo despite showing intact suppressive function in vitro. The seminal experiment from this era, which put the role of CTLA-4 in Treg function beyond doubt, was the demonstration by the Sakaguchi lab that mice selectively lacking CTLA-4 expression in Treg succumbed to a spontaneous lymphoproliferation with fatal $\mathrm{T}$ cell-dependent autoimmune disease[47]. In fact, mice lacking CTLA-4 in Treg only survived a few weeks longer than mice lacking CTLA-4 systemically. This marked a change in direction for the CTLA-4 field that, in some ways, was as significant as the original description of the knockout mice. It was now clear that a major role of CTLA-4 related to its ability to function in the regulatory T cell population. Indeed, transgenic expression of CTLA-4 was later shown 
to be one of three core requirements to convert a conventional CD4 T cell into a "homemade" regulatory T cell[48].

Another key message to arise from this era was the notion that there is a degree of redundancy in Treg suppressive mechanisms. For example it was shown that while wildtype Treg used CTLA-4 to suppress colitis, Treg lacking the CTLA-4 molecule were able to utilise IL-10 to achieve the same end[24]. Likewise, the function of CTLA-4 deficient Treg was shown to be partially TGF- $\beta$ dependent in a separate study[37], suggesting that, like IL-10, TGF- $\beta$ can also compensate to some extent for a lack of CTLA4. Together the experimental work indicated that the CTLA-4 pathway is an important component of Treg suppressive function, but that Treg also have other tools at their disposal to maintain immune homeostasis[49, 50]. It is likely, therefore, that the relative importance of any given suppressive mechanism varies depending on the physiological context of the immune response being regulated.

\section{CTLA-4 and the modification of APC phenotype}

Given that CTLA-4 appeared to comprise an important effector molecule for Treg suppression[51], this raised the question of how this might work at a molecular level. Models based on CTLA-4-dependent inhibition of TCR-mediated signaling were not useful in this context, since Treg require TCR signaling to elicit suppressive function[28, $52,53]$. A common theme that began to emerge from the literature was that CTLA-4expressing cells were able to modify the phenotype of antigen presenting cells. Early data suggested that this might reflect reverse-signaling by CD80/CD86 leading to 
activation of the enzyme indoleamine 2,3-dioxygenase (IDO) [54, 55]. However this hypothesis was challenged by transcriptional profiling analysis that failed to identify gene expression changes in CD80/CD86 expressing cells after treatment with soluble CTLA-4 fusion proteins (including abatacept and belatacept)[56]. Furthermore, it was shown that abatacept inhibits T cell proliferation without increasing expression of IDO[57], consistent with a role for CTLA-4 in limiting CD28 engagement via binding to their shared ligands, rather than triggering the IDO pathway. In addition, CTLA-4 blockade was even more effective in IDO deficient mice than wildtype mice[58], an observation that is hard to reconcile with the notion that CTLA-4 operates via IDO induction.

An independent observation that began to gain traction in the field was that Treg expressing CTLA-4 were able to downregulate expression of CD86 and CD80 on antigen presenting cells $[47,59-63]$. My group first noted this in experiments performed in vitro with murine Treg and splenic dendritic cells, where we observed a marked downregulation of CD86 (but not MHC class II) that could be inhibited by anti-CTLA-4 antibodies[40]. In collaboration with David Sansom's lab, across the hall, we were able to identify a molecular mechanism for the observed CTLA-4-mediated ligand downregulation. Through an extensive series of experiments in transfectant systems, primary cells, and ultimately in vivo models, we were able to show that CTLA-4 was capable of removing its ligands from adjacent cells by a process of transendocytosis[64]. Using our TCR-transgenic Treg system, we showed that Treg were able to capture GFP-tagged costimulatory ligands from APC in an antigen-dependent manner in vivo. Importantly, however CTLA-4 deficient Treg, which lacked regulatory capacity 
in the adoptive transfer model of diabetes[40], were unable to capture costimulatory ligands in vivo[64].

The description of CTLA-4-mediated trans-endocytosis changed our perspective on the entire biology of the CTLA-4 pathway. Suddenly the highly endocytic behaviour of the CTLA-4 molecule made sense - we now saw it as a molecular pump efficiently capturing ligands from the surface of opposing cells, trafficking them to lysosomes for degradation, then recycling to repeat. Similarly the sharing of ligands between CTLA-4 and CD28 seemed far more logical if the purpose of CTLA-4 was to strip APCs of costimulatory ligands, thereby limiting CD28 engagement, rather than to transmit inhibitory signals[65]. The conundrum of how ligands "decided" whether to positively signal via CD28 or negatively signal via CTLA-4 was instantly removed.

The notion that CTLA-4 functioned as a ligand competitor to regulate CD28 signaling was not new, and in fact was one of the earliest postulated mechanisms of action for CTLA-4 based on its superior affinity[66]. Trans-endocytosis effectively "supercharged" this concept: rather than costimulatory ligands being fleetingly blocked during a brief foray of a CTLA-4 molecule to the cell surface, instead CTLA-4 removed the ligands altogether from the APC and chaperoned them to lysosomes for degradation (Figure 4). Factor in the ability of the same CTLA-4 molecule to re-cycle to the surface for more, and we were now faced with a highly efficient system for limiting access of CD28 to costimulatory ligands.

Intriguingly, the key motif in the CTLA-4 cytoplasmic tail that confers endocytic behaviour, YVKM, is conserved in mammals and birds but not in species that are 
evolutionarily more primitive. Accordingly trout CTLA-4 is not subject to clathrinmediated endocytosis and predominantly exists as a cell surface protein[67]. It is therefore plausible that CTLA-4 originally operated in a cell-intrinsic manner, as a cell surface competitor to limit CD28 ligand binding, until the emergence of an intracellular trafficking motif took its function to the next level.

\section{Humans with CTLA-4 deficiency}

Perhaps the most unexpected development in our CTLA-4 journey was the fortuitous identification of patients with heterozgous CTLA-4 deficiency in our local CVID clinic, in collaboration with the Grimbacher and Sansom labs[68]. Of all the places we would have expected individuals with CTLA-4 deficiency to present, an immunodeficiency clinic was the last on the list. However, autoimmunity and immunodeficiency are strangely compatible bedfellows, both being manifestations of immune dysregulation. After all, normal immune homeostasis relies on a complex series of feedback mechanisms, with positive and negative regulation of the immune system being closely interlinked. Impaired immune responsiveness can compromise regulation (for example, reduced IL-2 production from conventional T cells impacts on Treg homeostasis) and conversely impaired Treg function can compromise protective immunity $[69,70]$. Epitomising this paradox, patients with CTLA-4 mutations exhibited diverse symptoms of autoimmunity (including autoimmune cytopenias, arthritis, thyroiditis, enteropathy and lymphadenopathy) yet showed a high incidence of hypogammaglobulinemia associated with a gradual loss of peripheral B cells. The link between hyperactivation of the T cell compartment and B cell attrition is currently unclear. 
Flow cytometric staining revealed that the Treg isolated from individuals with CTLA-4 mutations had decreased CTLA-4 expression and this was particularly marked following a brief period of in vitro restimulation. Furthermore, these Treg were impaired in their capacity to trans-endocytose costimulatory ligands and to elicit suppressive function in in vitro assays[68]. Analysis of an independent cohort of patients with CTLA-4 mutations at the NIH also revealed decreased CTLA-4 expression and reduced Treg function[71]. While the full clinical phenotype of this patient cohort is still emerging[68, 71-73], CTLA-4 heterozygous individuals have provided the first genetic evidence that CTLA-4 is an important component of Treg function and immune regulation in humans.

\section{CTLA-4 and Treg homeostasis}

In addition to contributing to Treg suppressive function, there is also evidence that CTLA-4 controls Treg homeostasis. We and others found that CTLA-4 knockout mice exhibit a dramatically expanded Treg population that show increased expression of the proliferation marker Ki67[40, 47]. This phenotype is recapitulated by inducible deletion of CTLA-4 in adult mice $[74,75]$ and in line with this, treatment of wildtype mice with blocking anti-CTLA-4 antibody augments Treg numbers in lymphoid tissues[76, 77]. Proliferation and expansion of Treg has also been reported in humans treated with antiCTLA-4 antibody[78] and Treg percentages are higher in some, but not all, individuals with CTLA-4 mutations [Hou et al Blood, in revision]. The fact that Treg can present in higher numbers in settings where they have been deprived of a key suppressive 
mechanism highlights that a simple assessment of Treg number can be a poor proxy for gauging the level of immune regulation.

The proliferation of Treg is known to be positively regulated by CD28 signaling $[44,45]$ suggesting a model in which CTLA-4 regulates Treg proliferation by controlling access of CD28 to their shared ligands (CD80 and CD86). This fits with clear evidence that the biological role of CTLA-4 is regulate the CD28 pathway: accordingly mice that lack CTLA4 but also lack CD28[43], or CD80/86[42], do not exhibit any signs of immune hyperactivation. Likewise, experimental blockade of CD80 and CD86 with a CTLA-4-Ig fusion protein or anti-CD80 and anti-CD86 antibodies also abrogates disease in CTLA-4deficient mice[37, 79]. It is known that Treg turn over to a higher extent than Tconv[46, 80-83], most likely reflecting their continuous encounter with self-antigens. Indeed antigen-specific Treg are known to accumulate in the lymph nodes draining the tissue site where their antigen is expressed[46], perhaps explaining why the Treg TCR repertoire varies by anatomical location[84]. Collectively, this indicates that local Treg homoestasis is shaped both by antigenic availability and by encounter with CD80/86 ligands, the latter being regulated by CTLA-4.

\section{Function of CTLA-4 in conventional T cells}

A considerable body of evidence, from both mouse and human, supported a major role for CTLA-4 in the Treg compartment. However, one conundrum that continued to trouble us was that CTLA-4 was known to function, albeit in a limited manner, in conventional T cells (Tconv) too. That being the case, why did CTLA-4 deficient Tconv behave identically to CTLA-4 sufficient Tconv in the mixed bone marrow chimeric mice 
discussed in section 1? Surely if CTLA-4 was operating in the Tconv compartment, there should be a discernable functional consequence of this. We reasoned that a potential explanation for this paradox could be that CTLA-4 operates in a cell-extrinsic, rather than cell-intrinsic, manner in Tconv.

To test this hypothesis, we set up a model in which OVA-specific conventional T cells (that were exclusively Foxp3-negative) were adoptively transferred into mice expressing OVA in the pancreas, allowing us to track the $\mathrm{T}$ cell response to antigen in the pancreatic lymph node. CTLA-4 deficient Tconv proliferated to a greater extent than CTLA-4 sufficient Tconv in this setting, highlighting that under certain conditions CTLA4 expressed in conventional $\mathrm{T}$ cells can regulate immune responsiveness. We next asked the key question - is CTLA-4 function in Tconv a cell-intrinsic or cell-extrinsic phenomenon? By co-transferring a mixture of wildtype and CTLA-4 deficient Tconv, identifiable on the basis of congenic markers, we were able to distinguish between these possibilities. Strikingly we found that CTLA-4-deficient Tconv no longer proliferated more than CTLA-4-sufficient Tconv if the two populations were co-transferred[85]. In fact, it appeared as though the CTLA-4-sufficient cells were "regulating" the CTLA-4 deficient ones. This regulatory behaviour was not associated with upregulation of the Treg-associated transcription factor Foxp3 in the T cells. Thus CTLA-4 appeared to operate in a cell-extrinsic "regulatory" manner, even when expressed in the conventional T cell compartment. Together with a similar study from the Alison lab[86], these findings resolved the paradox of why cell intrinsic functions of CTLA-4 are not apparent in mixed bone marrow chimeric mice. In other words, for both Treg and Tconv, CTLA-4 is not operating directly on the cell expressing it but instead is working indirectly such that CTLA-4+ cells modify the responses of CTLA-4- cells. Since Tconv, 
like Treg, can use CTLA-4 to perform trans-endocytosis[64], it seems likely that CTLA-4 operates by modifying APC phenotype regardless of the cell type it is expressed in.

Of note, while CTLA-4 is clearly able to operate in the conventional T cell compartment, all of our experience suggests the major function of CTLA-4 is in Treg. In fact, to reveal the function Tconv-expressed CTLA-4 in vivo it is often necessary to remove or inactivate Treg, since the dominant role of CTLA-4 in this population can mask its more subtle role in Tconv. It is tempting to speculate that the evolution of Foxp3 and Treg cells[87, 88], coincident with the evolution of CTLA-4 endocytosis, permitted a previously cell-intrinsic mechanism of regulation to be outsourced to dedicated cell population for use in a cell-extrinsic manner. As a result, Treg wield CTLA-4 like true professionals and are the dominant vehicle for CTLA-4-mediated immune regulation, although, if need be, conventional T cells can still employ it to temper clone size.

\section{CTLA-4 regulates follicular helper $\mathrm{T}$ cell differentiation}

In the trans-endocytosis model, CTLA-4-dependent removal of costimulatory ligands from APC serves to limit T cell CD28 engagement. Thus, the key downstream consequence of CTLA-4 function is a quantitative alteration of CD28 signaling. In this view, CD28 knockout and CTLA-4 knockout mice represent two extreme ends of the same spectrum, presenting phenotypes of defective and excessive CD28 triggering respectively. In this regard, we recently noted that T cells in CTLA-4-deficient mice undergo spontaneous differentiation into follicular helper T cells (Tfh), a subset known to be dependent on CD28 signaling. Tfh cells support the fine-tuning of B cell antibody 
responses within specialised structures termed germinal centers and it has been shown previously that CD28 deficient T cells are unable to differentiate into Tfh[89] and support germinal center formation[90, 91]. The overproduction of Tfh in CTLA-4 deficient mice extends these observations by suggesting that increasing the level of CD28 engagement triggers a quantitative increase in Tfh differentiation. In other words, it's not simply that CD28 is needed for Tfh, but instead the amount of CD28 engagement is directly coupled to the extent of Tfh differentation. Consistent with this notion, increasing CD28 engagement (by blocking CTLA-4) was sufficient to trigger spontaneous Tfh differentiation in wildtype mice: conversely, limiting CD28 engagement by using naive T cells from a CD28 heterozygous donor resulted in a marked diminution of $\mathrm{Tfh}$ differentiation[92]. Our own studies did not address which cell type CTLA-4 had to be expressed on in order to control CD28-mediated Tfh differentiation. However work from the Sakaguchi[93] and Sharpe[94] labs has revealed that deletion of CTLA-4 from the Treg population is sufficient to trigger increased Tfh differentiation, consistent with the idea that the major function of CTLA-4 maps to this population. Taken together, these experiments suggest that the ability of CTLA-4 to regulate CD28 engagement on conventional T cells not only dictates whether or not those T cells will become activated, but also has implications for the fate decisions of the T cells post activation, notably their propensity to differentiate into Tfh.

\section{Concluding remarks}

A major early research focus for my group was dissecting how Treg inhibit autoimmune pathology in a mouse model of diabetes. This led to the observation that CTLA-4 
expression was required in the Treg population for effective control of T cell-mediated pancreatic islet destruction. Our search for the molecular mechanism underlying this observation sparked a highly productive (and ongoing) collaboration with the Sansom group in which we identified trans-endocytosis of costimulatory ligands as a mechanism of action for CTLA-4. By limiting interaction of CD28 with its ligands, CTLA-4 exerts control over all CD28-dependent functions including Tfh differentiation which is highly sensitive to the level of CD28 engagement[92]. Since dysregulated Tfh differentiation is increasingly associated with autoimmune outcomes[95-98], regulation of this process represents another major mechanism by which CTLA-4 protects from autoimmunity. Thus CTLA-4 serves as a key effector molecule in Treg where its actions both set the threshold for activation of naive T cells and influence the differentiation, and thereby pathogenicity, of T cells following activation.

\section{Acknowledgements}

I would like to acknowledge the many generous collaborators who made my early experiments possible, in particular Arlene Sharpe who kindly gifted her CTLA-4deficient mice. The work of the author is currently funded by The Medical Research Council, Diabetes UK and The Rosetrees Trust. The author has received funding under the European Union's Horizon 2020 research and innovation programme under the Marie Sklodowska-Curie grant agreement No 675395 and holds a Royal Society Wolfson Research Merit Award. I am grateful to Vitalijs Ovcinnikovs for helpful comments on the manuscript. 


\section{References}

[1] L.S. Walker, D.M. Sansom, The emerging role of CTLA4 as a cell-extrinsic regulator of T cell responses, Nat Rev Immunol 11(12) (2011) 852-63.

[2] A. Shahinian, K. Pfeffer, K.P. Lee, T.M. Kundig, K. Kishihara, A. Wakeham, K. Kawai, P.S. Ohashi, C.B. Thompson, T.W. Mak, Differential T cell costimulatory requirements in CD28-deficient mice, Science 261(5121) (1993) 609-12.

[3] M.E. Keir, A.H. Sharpe, The B7/CD28 costimulatory family in autoimmunity, Immunol Rev 204 (2005) 128-43.

[4] E.A. Tivol, F. Borriello, A.N. Schweitzer, W.P. Lynch, J.A. Bluestone, A.H. Sharpe, Loss of CTLA-4 leads to massive lymphoproliferation and fatal multiorgan tissue destruction, revealing a critical negative regulatory role of CTLA-4, Immunity 3(5) (1995) 541-7. [5] P. Waterhouse, J.M. Penninger, E. Timms, A. Wakeham, A. Shahinian, K.P. Lee, C.B. Thompson, H. Griesser, T.W. Mak, Lymphoproliferative disorders with early lethality in mice deficient in Ctla-4, Science 270(5238) (1995) 985-8.

[6] T. Shiratori, S. Miyatake, H. Ohno, C. Nakaseko, K. Isono, J.S. Bonifacino, T. Saito, Tyrosine phosphorylation controls internalization of CTLA-4 by regulating its interaction with clathrin-associated adaptor complex AP-2, Immunity 6 (1997) 583-589. [7] E. Chuang, M.L. Alegre, C.S. Duckett, P.J. Noel, M.G. Vander Heiden, C.B. Thompson, Interaction of CTLA-4 with the clathrin-associated protein AP50 results in ligandindependent endocytosis that limits cell surface expression, J Immunol 159(1) (1997) 144-51.

[8] Y. Zhang, J.P. Allison, Interaction of CTLA-4 with AP-50, a clathrin-coated pit adaptor protein, Proc.Natl.Acad.Sci.USA 94 (1997) 9273-9278. 
[9] R.H. Schwartz, A cell culture model for T lymphocyte clonal anergy, Science 248(4961) (1990) 1349-56.

[10] J.P. Allison, CD28-B7 interactions in T-cell activation, Curr Opin Immunol 6(3) (1994) 414-9.

[11] P. Lane, C. Haller, F. McConnell, Evidence that induction of tolerance in vivo involves active signaling via a B7 ligand-dependent mechanism: CTLA4-Ig protects V beta 8+ T cells from tolerance induction by the superantigen staphylococcal enterotoxin B, Eur J Immunol 26(4) (1996) 858-62.

[12] V.L. Perez, L. Van Parijs, A. Biuckians, X.X. Zheng, T.B. Strom, A.K. Abbas, Induction of peripheral T cell tolerance in vivo requires CTLA-4 engagement, Immunity 6(4) (1997) 411-7.

[13] A.D. Wells, M.C. Walsh, J.A. Bluestone, L.A. Turka, Signaling through CD28 and CTLA4 controls two distinct forms of T cell anergy, J Clin Invest 108(6) (2001) 895-903. [14] M.F. Krummel, J.P. Allison, CD28 and CTLA-4 have opposing effects on the response of T cells to stimulation, J Exp Med 182(2) (1995) 459-65.

[15] T.L. Walunas, D.J. Lenschow, C.Y. Bakker, P.S. Linsley, G.J. Freeman, J.M. Green, C.B. Thompson, J.A. Bluestone, CTLA-4 can function as a negative regulator of T cell activation, Immunity 1(5) (1994) 405-13.

[16] M.F. Krummel, J.P. Allison, CTLA-4 engagement inhibits IL-2 accumulation and cell cycle progression upon activation of resting T cells, J.Exp.Med. 183 (1996) 2533-2540. [17] T.L. Walunas, C.Y. Bakker, J.A. Bluestone, CTLA-4 ligation blocks CD28-dependent T cell activation, J.Exp.Med. 183 (1996) 2541-2550.

[18] J.P. Allison, M.F. Krummel, The Yin and Yang of T cell costimulation, Science $270(5238)(1995)$ 932-3. 
[19] L.E. Marengere, P. Waterhouse, G.S. Duncan, H.W. Mittrucker, G.S. Feng, T.W. Mak, Regulation of T cell receptor signaling by tyrosine phosphatase SYP association with CTLA-4, Science 272(5265) (1996) 1170-3.

[20] K.M. Lee, E. Chuang, M. Griffin, R. Khattri, D.K. Hong, W. Zhang, D. Straus, L.E. Samelson, C.B. Thompson, J.A. Bluestone, Molecular basis of T cell inactivation by CTLA4, Science 282(5397) (1998) 2263-6.

[21] M.F. Bachmann, G. Kohler, B. Ecabert, T.W. Mak, M. Kopf, Cutting edge: lymphoproliferative disease in the absence of CTLA-4 is not T cell autonomous, J Immunol 163(3) (1999) 1128-31.

[22] D. Homann, W. Dummer, T. Wolfe, E. Rodrigo, A.N. Theofilopoulos, M.B. Oldstone, M.G. von Herrath, Lack of intrinsic CTLA-4 expression has minimal effect on regulation of antiviral T-cell immunity, J Virol 80(1) (2006) 270-80.

[23] M.F. Bachmann, A. Gallimore, E. Jones, B. Ecabert, H. Acha-Orbea, M. Kopf, Normal pathogen-specific immune responses mounted by CTLA-4-deficient T cells: a paradigm reconsidered, Eur J Immunol 31(2) (2001) 450-8.

[24] S. Read, R. Greenwald, A. Izcue, N. Robinson, D. Mandelbrot, L. Francisco, A.H.

Sharpe, F. Powrie, Blockade of CTLA-4 on CD4+CD25+ regulatory T cells abrogates their function in vivo, J Immunol 177(7) (2006) 4376-83.

[25] S. Chikuma, J.A. Bluestone, Expression of CTLA-4 and FOXP3 in cis protects from lethal lymphoproliferative disease, Eur J Immunol 37(5) (2007) 1285-9.

[26] R.H. Friedline, D.S. Brown, H. Nguyen, H. Kornfeld, J. Lee, Y. Zhang, M. Appleby, S.D. Der, J. Kang, C.A. Chambers, CD4+ regulatory T cells require CTLA-4 for the maintenance of systemic tolerance, J Exp Med 206(2) (2009) 421-34. 
[27] X. Tai, F. Van Laethem, L. Pobezinsky, T. Guinter, S.O. Sharrow, A. Adams, L. Granger, M. Kruhlak, T. Lindsten, C.B. Thompson, L. Feigenbaum, A. Singer, Basis of CTLA-4 function in regulatory and conventional CD4(+) T cells, Blood 119(22) (2012) 5155-63. [28] T. Takahashi, Y. Kuniyasu, M. Toda, N. Sakaguchi, M. Itoh, M. Iwata, J. Shimizu, S. Sakaguchi, Immunologic self-tolerance maintained by CD25+CD4+ naturally anergic and suppressive T cells: induction of autoimmune disease by breaking their anergic/suppressive state, Int Immunol 10(12) (1998) 1969-80.

[29] A.M. Thornton, E.M. Shevach, CD4+CD25+ immunoregulatory T cells suppress polyclonal T cell activation in vitro by inhibiting interleukin 2 production, J Exp Med 188(2) (1998) 287-96.

[30] S. Hori, T. Nomura, S. Sakaguchi, Control of regulatory T cell development by the transcription factor Foxp3, Science 299(5609) (2003) 1057-61.

[31] J.D. Fontenot, M.A. Gavin, A.Y. Rudensky, Foxp3 programs the development and function of CD4+CD25+ regulatory T cells, Nat Immunol 4(4) (2003) 330-6.

[32] R. Khattri, T. Cox, S.A. Yasayko, F. Ramsdell, An essential role for Scurfin in CD4+CD25+ T regulatory cells, Nat Immunol 4(4) (2003) 337-42.

[33] R.S. Wildin, F. Ramsdell, J. Peake, F. Faravelli, J.L. Casanova, N. Buist, E. Levy-Lahad, M. Mazzella, O. Goulet, L. Perroni, F.D. Bricarelli, G. Byrne, M. McEuen, S. Proll, M. Appleby, M.E. Brunkow, X-linked neonatal diabetes mellitus, enteropathy and endocrinopathy syndrome is the human equivalent of mouse scurfy, Nat Genet 27(1) (2001) 18-20.

[34] C.L. Bennett, J. Christie, F. Ramsdell, M.E. Brunkow, P.J. Ferguson, L. Whitesell, T.E. Kelly, F.T. Saulsbury, P.F. Chance, H.D. Ochs, The immune dysregulation, polyendocrinopathy, enteropathy, X-linked syndrome (IPEX) is caused by mutations of FOXP3, Nat Genet 27(1) (2001) 20-1. 
[35] S. Read, V. Malmstrom, F. Powrie, Cytotoxic T lymphocyte-associated antigen 4 plays an essential role in the function of $\mathrm{CD} 25(+) \mathrm{CD} 4(+)$ regulatory cells that control intestinal inflammation, J Exp Med 192(2) (2000) 295-302.

[36] T. Takahashi, T. Tagami, S. Yamazaki, T. Uede, J. Shimizu, N. Sakaguchi, T.W. Mak, S. Sakaguchi, Immunologic self-tolerance maintained by CD25(+)CD4(+) regulatory T cells constitutively expressing cytotoxic T lymphocyte-associated antigen 4, J Exp Med 192(2) (2000) 303-10.

[37] Q. Tang, E.K. Boden, K.J. Henriksen, H. Bour-Jordan, M. Bi, J.A. Bluestone, Distinct roles of CTLA-4 and TGF-beta in CD4+CD25+ regulatory T cell function, Eur J Immunol 34(11) (2004) 2996-3005.

[38] A.M. Thornton, C.A. Piccirillo, E.M. Shevach, Activation requirements for the induction of CD4+CD25+ T cell suppressor function, Eur J Immunol 34(2) (2004) 36676.

[39] D.K. Sojka, A. Hughson, D.J. Fowell, CTLA-4 is required by CD4+CD25+ Treg to control CD4+ T-cell lymphopenia-induced proliferation, Eur J Immunol 39(6) (2009) 1544-51.

[40] E.M. Schmidt, C.J. Wang, G.A. Ryan, L.E. Clough, O.S. Qureshi, M. Goodall, A.K. Abbas, A.H. Sharpe, D.M. Sansom, L.S. Walker, CTLA-4 controls regulatory T cell peripheral homeostasis and is required for suppression of pancreatic islet autoimmunity, J Immunol 182(1) (2009) 274-82.

[41] E.A. Tivol, S.D. Boyd, S. McKeon, F. Borriello, P. Nickerson, T.B. Strom, A.H. Sharpe, CTLA4Ig prevents lymphoproliferation and fatal multiorgan tissue destruction in CTLA4-deficient mice, J Immunol 158(11) (1997) 5091-4. 
[42] D.A. Mandelbrot, A.J. McAdam, A.H. Sharpe, B7-1 or B7-2 is required to produce the lymphoproliferative phenotype in mice lacking cytotoxic T lymphocyte-associated antigen 4 (CTLA-4), J.Exp.Med. 189 (1999) 435-440.

[43] X. Tai, F. Van Laethem, A.H. Sharpe, A. Singer, Induction of autoimmune disease in CTLA-4-/- mice depends on a specific CD28 motif that is required for in vivo costimulation, Proc Natl Acad Sci U S A 104(34) (2007) 13756-61.

[44] B. Salomon, D.J. Lenschow, L. Rhee, N. Ashourian, B. Singh, A. Sharpe, J.A. Bluestone, B7/CD28 costimulation is essential for the homeostasis of the CD4+CD25+ immunoregulatory T cells that control autoimmune diabetes, Immunity 12(4) (2000) 431-40.

[45] Q. Tang, K.J. Henriksen, E.K. Boden, A.J. Tooley, J. Ye, S.K. Subudhi, X.X. Zheng, T.B. Strom, J.A. Bluestone, Cutting edge: CD28 controls peripheral homeostasis of CD4+CD25+ regulatory T cells, J Immunol 171(7) (2003) 3348-52.

[46] L.S. Walker, A. Chodos, M. Eggena, H. Dooms, A.K. Abbas, Antigen-dependent proliferation of CD4+ CD25+ regulatory T cells in vivo, J Exp Med 198(2) (2003) 249-58. [47] K. Wing, Y. Onishi, P. Prieto-Martin, T. Yamaguchi, M. Miyara, Z. Fehervari, T. Nomura, S. Sakaguchi, CTLA-4 control over Foxp3+ regulatory T cell function, Science 322(5899) (2008) 271-5.

[48] T. Yamaguchi, A. Kishi, M. Osaki, H. Morikawa, P. Prieto-Martin, K. Wing, T. Saito, S. Sakaguchi, Construction of self-recognizing regulatory T cells from conventional T cells by controlling CTLA-4 and IL-2 expression, Proceedings of the National Academy of Sciences of the United States of America 110(23) (2013) E2116-25. [49] D.A. Vignali, L.W. Collison, C.J. Workman, How regulatory T cells work, Nat Rev Immunol 8(7) (2008) 523-32. 
[50] Q. Tang, J.A. Bluestone, The Foxp3+ regulatory T cell: a jack of all trades, master of regulation, Nat Immunol 9(3) (2008) 239-44.

[51] L.S. Walker, Treg and CTLA-4: Two intertwining pathways to immune tolerance, J Autoimmun 45 (2013) 49-57.

[52] A.M. Thornton, E.M. Shevach, Suppressor effector function of CD4+CD25+ immunoregulatory T cells is antigen nonspecific, J Immunol 164(1) (2000) 183-90. [53] A.G. Levine, A. Arvey, W. Jin, A.Y. Rudensky, Continuous requirement for the TCR in regulatory T cell function, Nat Immunol 15(11) (2014) 1070-8.

[54] U. Grohmann, C. Orabona, F. Fallarino, C. Vacca, F. Calcinaro, A. Falorni, P.

Candeloro, M.L. Belladonna, R. Bianchi, M.C. Fioretti, P. Puccetti, CTLA-4-Ig regulates tryptophan catabolism in vivo, Nat Immunol 3(11) (2002) 1097-101.

[55] F. Fallarino, U. Grohmann, K.W. Hwang, C. Orabona, C. Vacca, R. Bianchi, M.L. Belladonna, M.C. Fioretti, M.L. Alegre, P. Puccetti, Modulation of tryptophan catabolism by regulatory T cells, Nat Immunol 4(12) (2003) 1206-12.

[56] J.A. Carman, P.M. Davis, W.P. Yang, J. Zhu, H. Chang, A. He, A. Truong, S.J. Suchard, S.G. Nadler, Abatacept does not induce direct gene expression changes in antigenpresenting cells, J Clin Immunol 29(4) (2009) 479-89.

[57] P.M. Davis, S.G. Nadler, D.K. Stetsko, S.J. Suchard, Abatacept modulates human dendritic cell-stimulated T-cell proliferation and effector function independent of IDO induction, Clin Immunol 126(1) (2008) 38-47.

[58] R.B. Holmgaard, D. Zamarin, D.H. Munn, J.D. Wolchok, J.P. Allison, Indoleamine 2,3dioxygenase is a critical resistance mechanism in antitumor T cell immunotherapy targeting CTLA-4, J Exp Med 210(7) (2013) 1389-402. 
[59] L. Cederbom, H. Hall, F. Ivars, CD4+CD25+ regulatory T cells down-regulate costimulatory molecules on antigen-presenting cells, Eur J Immunol 30(6) (2000) 153843.

[60] P. Serra, A. Amrani, J. Yamanouchi, B. Han, S. Thiessen, T. Utsugi, J. Verdaguer, P. Santamaria, CD40 ligation releases immature dendritic cells from the control of regulatory CD4+CD25+ T cells, Immunity 19(6) (2003) 877-89.

[61] N. Misra, J. Bayry, S. Lacroix-Desmazes, M.D. Kazatchkine, S.V. Kaveri, Cutting edge: human CD4+CD25+ T cells restrain the maturation and antigen-presenting function of dendritic cells, J Immunol 172(8) (2004) 4676-80.

[62] C. Oderup, L. Cederbom, A. Makowska, C.M. Cilio, F. Ivars, Cytotoxic T lymphocyte antigen-4-dependent down-modulation of costimulatory molecules on dendritic cells in CD4+ CD25+ regulatory T-cell-mediated suppression, Immunology 118(2) (2006) 2409.

[63] Y. Onishi, Z. Fehervari, T. Yamaguchi, S. Sakaguchi, Foxp3+ natural regulatory T cells preferentially form aggregates on dendritic cells in vitro and actively inhibit their maturation, Proc Natl Acad Sci U S A 105(29) (2008) 10113-8.

[64] O.S. Qureshi, Y. Zheng, K. Nakamura, K. Attridge, C. Manzotti, E.M. Schmidt, J. Baker, L.E. Jeffery, S. Kaur, Z. Briggs, T.Z. Hou, C.E. Futter, G. Anderson, L.S. Walker, D.M. Sansom, Trans-endocytosis of CD80 and CD86: a molecular basis for the cell-extrinsic function of CTLA-4, Science 332(6029) (2011) 600-3.

[65] L.S. Walker, D.M. Sansom, Confusing signals: recent progress in CTLA-4 biology, Trends Immunol 36(2) (2015) 63-70.

[66] C.B. Thompson, J.P. Allison, The emerging role of CTLA-4 as an immune attenuator, Immunity 7(4) (1997) 445-50. 
[67] S. Kaur, O.S. Qureshi, D.M. Sansom, Comparison of the intracellular trafficking itinerary of ctla-4 orthologues, PLoS One 8(4) (2013) e60903.

[68] D. Schubert, C. Bode, R. Kenefeck, T.Z. Hou, J.B. Wing, A. Kennedy, A. Bulashevska, B.S. Petersen, A.A. Schaffer, B.A. Gruning, S. Unger, N. Frede, U. Baumann, T. Witte, R.E. Schmidt, G. Dueckers, T. Niehues, S. Seneviratne, M. Kanariou, C. Speckmann, S. Ehl, A. Rensing-Ehl, K. Warnatz, M. Rakhmanov, R. Thimme, P. Hasselblatt, F. Emmerich, T. Cathomen, R. Backofen, P. Fisch, M. Seidl, A. May, A. Schmitt-Graeff, S. Ikemizu, U. Salzer, A. Franke, S. Sakaguchi, L.S. Walker, D.M. Sansom, B. Grimbacher, Autosomal dominant immune dysregulation syndrome in humans with CTLA4 mutations, Nature Medicine $20(12)(2014) 1410-1416$.

[69] J.M. Lund, L. Hsing, T.T. Pham, A.Y. Rudensky, Coordination of early protective immunity to viral infection by regulatory T cells, Science 320(5880) (2008) 1220-4. [70] K.A. Smith, K.J. Filbey, L.A. Reynolds, J.P. Hewitson, Y. Harcus, L. Boon, T.

Sparwasser, G. Hammerling, R.M. Maizels, Low-level regulatory T-cell activity is essential for functional type-2 effector immunity to expel gastrointestinal helminths, Mucosal Immunol 9(2) (2016) 428-43.

[71] H.S. Kuehn, W. Ouyang, B. Lo, E.K. Deenick, J.E. Niemela, D.T. Avery, J.N. Schickel, D.Q. Tran, J. Stoddard, Y. Zhang, D.M. Frucht, B. Dumitriu, P. Scheinberg, L.R. Folio, C.A. Frein, S. Price, C. Koh, T. Heller, C.M. Seroogy, A. Huttenlocher, V.K. Rao, H.C. Su, D. Kleiner, L.D. Notarangelo, Y. Rampertaap, K.N. Olivier, J. McElwee, J. Hughes, S. Pittaluga, J.B. Oliveira, E. Meffre, T.A. Fleisher, S.M. Holland, M.J. Lenardo, S.G. Tangye, G. Uzel, Immune dysregulation in human subjects with heterozygous germline mutations in CTLA4, Science 345(6204) (2014) 1623-7.

[72] S. Zeissig, B.S. Petersen, M. Tomczak, E. Melum, E. Huc-Claustre, S.K. Dougan, J.K. Laerdahl, B. Stade, M. Forster, S. Schreiber, D. Weir, A.M. Leichtner, A. Franke, R.S. 
Blumberg, Early-onset Crohn's disease and autoimmunity associated with a variant in CTLA-4, Gut 64(12) (2015) 1889-97.

[73] S. Hayakawa, S. Okada, M. Tsumura, S. Sakata, Y. Ueno, K. Imai, T. Morio, O. Ohara, K. Chayama, M. Kobayashi, A Patient with CTLA-4 Haploinsufficiency Presenting Gastric Cancer, J Clin Immunol 36(1) (2016) 28-32.

[74] A.M. Paterson, S.B. Lovitch, P.T. Sage, V.R. Juneja, Y. Lee, J.D. Trombley, C.V. Arancibia-Carcamo, R.A. Sobel, A.Y. Rudensky, V.K. Kuchroo, G.J. Freeman, A.H. Sharpe, Deletion of CTLA-4 on regulatory T cells during adulthood leads to resistance to autoimmunity, J Exp Med 212(10) (2015) 1603-21.

[75] K. Klocke, S. Sakaguchi, R. Holmdahl, K. Wing, Induction of autoimmune disease by deletion of CTLA-4 in mice in adulthood, Proc Natl Acad Sci U S A 113(17) (2016) E2383-92.

[76] S.A. Quezada, K.S. Peggs, M.A. Curran, J.P. Allison, CTLA4 blockade and GM-CSF combination immunotherapy alters the intratumor balance of effector and regulatory $\mathrm{T}$ cells, J Clin Invest 116(7) (2006) 1935-45.

[77] A.L. Tang, J.R. Teijaro, M.N. Njau, S.S. Chandran, A. Azimzadeh, S.G. Nadler, D.M. Rothstein, D.L. Farber, CTLA4 expression is an indicator and regulator of steady-state CD4+ FoxP3+ T cell homeostasis, J Immunol 181(3) (2008) 1806-13.

[78] B. Kavanagh, S. O'Brien, D. Lee, Y. Hou, V. Weinberg, B. Rini, J.P. Allison, E.J. Small, L. Fong, CTLA4 blockade expands FoxP3+ regulatory and activated effector CD4+ T cells in a dose-dependant fashion, Blood (2008).

[79] C.A. Chambers, T.J. Sullivan, J.P. Allison, Lymphoproliferation in CTLA-4-deficient mice is mediated by costimulation-dependent activation of CD4+ cells, Immunity 7 (1997) 885-895. 
[80] S. Fisson, G. Darrasse-Jeze, E. Litvinova, F. Septier, D. Klatzmann, R. Liblau, B.L. Salomon, Continuous activation of autoreactive CD4+ CD25+ regulatory T cells in the steady state, J Exp Med 198(5) (2003) 737-46.

[81] L. Klein, K. Khazaie, H. von Boehmer, In vivo dynamics of antigen-specific regulatory T cells not predicted from behavior in vitro, Proc Natl Acad Sci U S A 100(15) (2003) 8886-91.

[82] L.S. Walker, CD4+ CD25+ Treg: divide and rule?, Immunology 111(2) (2004) 12937.

[83] K. Attridge, L.S. Walker, Homeostasis and function of regulatory T cells (Tregs) in vivo: lessons from TCR-transgenic Tregs, Immunological reviews 259(1) (2014) 23-39. [84] S.K. Lathrop, N.A. Santacruz, D. Pham, J. Luo, C.S. Hsieh, Antigen-specific peripheral shaping of the natural regulatory T cell population, J Exp Med 205(13) (2008) 3105-17. [85] C.J. Wang, R. Kenefeck, L. Wardzinski, K. Attridge, C. Manzotti, E.M. Schmidt, O.S. Qureshi, D.M. Sansom, L.S. Walker, Cutting edge: cell-extrinsic immune regulation by ctla-4 expressed on conventional T cells, Journal of Immunology 189(3) (2012) 1118-22. [86] E. Corse, J.P. Allison, Cutting edge: CTLA-4 on effector T cells inhibits in trans, Journal of Immunology 189(3) (2012) 1123-7.

[87] K.G. Andersen, J.K. Nissen, A.G. Betz, Comparative Genomics Reveals Key Gain-ofFunction Events in Foxp3 during Regulatory T Cell Evolution, Front Immunol 3 (2012) 113.

[88] M.P. Denyer, D.Y. Pinheiro, O.A. Garden, A.J. Shepherd, Missed, Not Missing: Phylogenomic Evidence for the Existence of Avian FoxP3, PLoS One 11(3) (2016) e0150988.

[89] L.S. Walker, A. Gulbranson-Judge, S. Flynn, T. Brocker, C. Raykundalia, M. Goodall, R. Forster, M. Lipp, P. Lane, Compromised OX40 function in CD28-deficient mice is linked 
with failure to develop CXCR5-positive CD4 cells and germinal centers, J Exp Med 190(8) (1999) 1115-22.

[90] S.E. Ferguson, S. Han, G. Kelsoe, C.B. Thompson, CD28 is required for germinal center formation, J Immunol 156(12) (1996) 4576-81.

[91] F. Borriello, M.P. Sethna, S.D. Boyd, A.N. Schweitzer, E.A. Tivol, D. Jacoby, T.B. Strom, E.M. Simpson, G.J. Freeman, A.H. Sharpe, B7-1 and B7-2 have overlapping, critical roles in immunoglobulin class switching and germinal center formation, Immunity 6 (1997) 303-313.

[92] C.J. Wang, F. Heuts, V. Ovcinnikovs, L. Wardzinski, C. Bowers, E.M. Schmidt, A. Kogimtzis, R. Kenefeck, D.M. Sansom, L.S. Walker, CTLA-4 controls follicular helper T-cell differentiation by regulating the strength of CD28 engagement, Proc Natl Acad Sci U S A 112(2) (2015) 524-9.

[93] J.B. Wing, W. Ise, T. Kurosaki, S. Sakaguchi, Regulatory T cells control antigenspecific expansion of Tfh cell number and humoral immune responses via the coreceptor CTLA-4, Immunity 41(6) (2014) 1013-25.

[94] P.T. Sage, A.M. Paterson, S.B. Lovitch, A.H. Sharpe, The coinhibitory receptor CTLA-4 controls B cell responses by modulating $\mathrm{T}$ follicular helper, $\mathrm{T}$ follicular regulatory, and $\mathrm{T}$ regulatory cells, Immunity 41(6) (2014) 1026-39.

[95] M.A. Linterman, R.J. Rigby, R.K. Wong, D. Yu, R. Brink, J.L. Cannons, P.L. Schwartzberg, M.C. Cook, G.D. Walters, C.G. Vinuesa, Follicular helper T cells are required for systemic autoimmunity, The Journal of Experimental Medicine 206(3) (2009) 56176.

[96] S.G. Tangye, C.S. Ma, R. Brink, E.K. Deenick, The good, the bad and the ugly - TFH cells in human health and disease, Nature Reviews Immunology 13(6) (2013) 412-26. 
[97] R. Kenefeck, C.J. Wang, T. Kapadi, L. Wardzinski, K. Attridge, L.E. Clough, F. Heuts, A. Kogimtzis, S. Patel, M. Rosenthal, M. Ono, D.M. Sansom, P. Narendran, L.S. Walker, Follicular helper T cell signature in type 1 diabetes, Journal of Clinical Investigation 125(1) (2015) 292-303.

[98] H. Ueno, T follicular helper cells in human autoimmunity, Curr Opin Immunol 43 (2016) 24-31. 


\section{Figure Legends}

\section{Figure 1. CD28 and CTLA-4: T cell proteins with shared ligands and opposing}

functions. CD28 is expressed at the T cell surface and CTLA-4 is predominantly intracellular. CTLA-4 is constitutively expressed on regulatory T cells and induced upon activation in conventional T cells. CD28 and CTLA-4 bind to the same ligands, CD80 and CD86, expressed on antigen presenting cells.

\section{Figure 2. Cell-extrinsic function of CTLA-4 revealed by mixed bone marrow} chimeric mice. Rag-/- deficient mice were reconstituted with bone marrow from wildtype mice, CTLA4-/- mice, or a 1:1 mixture of both. CTLA-4-/- cells exhibit hyperactivation and lymphoproliferation (red) when alone, but remain non-activated (green) when in the presence of wildtype cells. Observation first reported in ref 21.

Figure 3. CTLA-4-/- antigen-specific Treg fail to control islet autoimmunity. i) Diabetes was induced by adoptively transferring OVA-specific T cells into rag2-/- mice expressing OVA in pancreatic beta-cells. ii) Co-transfer of OVA-specific Treg (isolated from D011xrip-mOVA/rag-/- mice) protected from diabetes. iii) Co-transfer of CTLA4deficient OVA-specific Treg (isolated from D011xrip-m0VA/ctla4-/-/rag-/- mice) failed to confer disease protection (see ref 40 ).

Figure 4. CTLA-4-mediated trans-endocytosis removes costimulatory ligands from APC. i) In the absence of Treg, APC can provide costimulatory ligands to activate Tconv. ii) Treg-expressed CTLA-4 binds to costimulatory ligands (CD80/86) and iii) removes 
them from the APC. This APC is now unable to provide costimulatory signals to activate Tconv. 


\section{Highlights}

- CTLA-4 is a critical negative regulator of the T cell immune responses

- CTLA-4 can function in a cell-extrinsic manner

- CTLA-4 is required for regulatory $\mathrm{T}$ cell function in certain settings

- CTLA-4 can downregulate its ligands by a process of transendocytosis

- By controlling follicular helper T cell differentiation, CTLA4 can regulate B-cell as well as $\mathrm{T}$-cell responses 


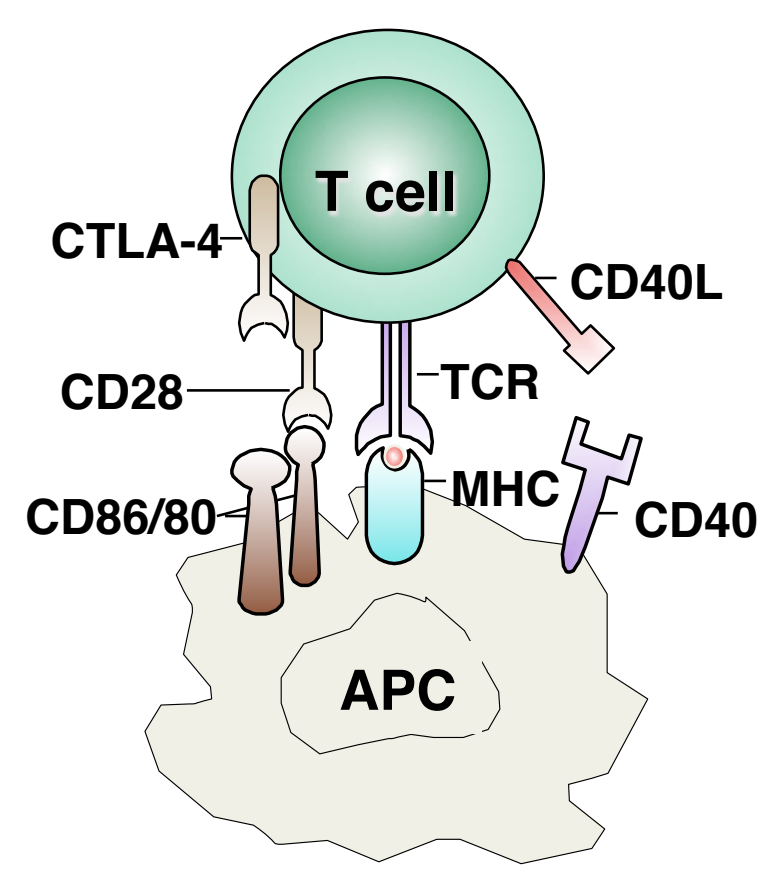

Figure 1. CD28 and CTLA-4: T cell proteins with shared ligands and opposing functions. CD28 is expressed at the $T$ cell surface and CTLA-4 is predominantly intracellular. CTLA-4 is constitutively expressed on regulatory $T$ cells and induced upon activation in conventional T cells. CD28 and CTLA-4 bind to the same ligands, CD80 and CD86, expressed on antigen presenting cells. 


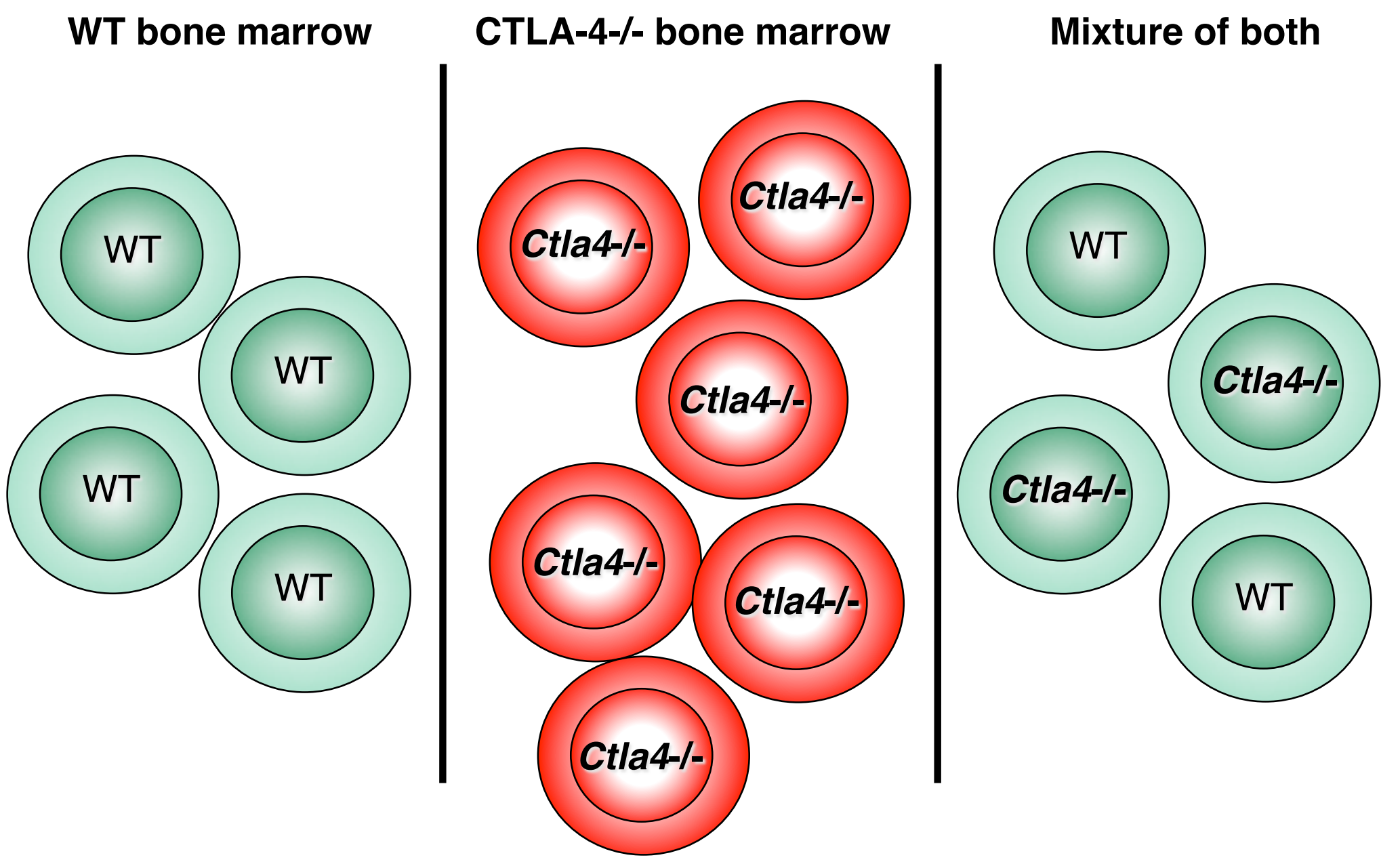

Figure 2. Cell-extrinsic function of CTLA-4 revealed by mixed bone marrow chimeric mice. Rag-/- deficient mice were reconstituted with bone marrow from wildtype mice, CTLA4-/mice, or a 1:1 mixture of both. CTLA-4-/- cells exhibit hyperactivation and lymphoproliferation (red) when alone, but remain non-activated (green) when in the presence of wildtype cells. Observation first reported in ref 21. 


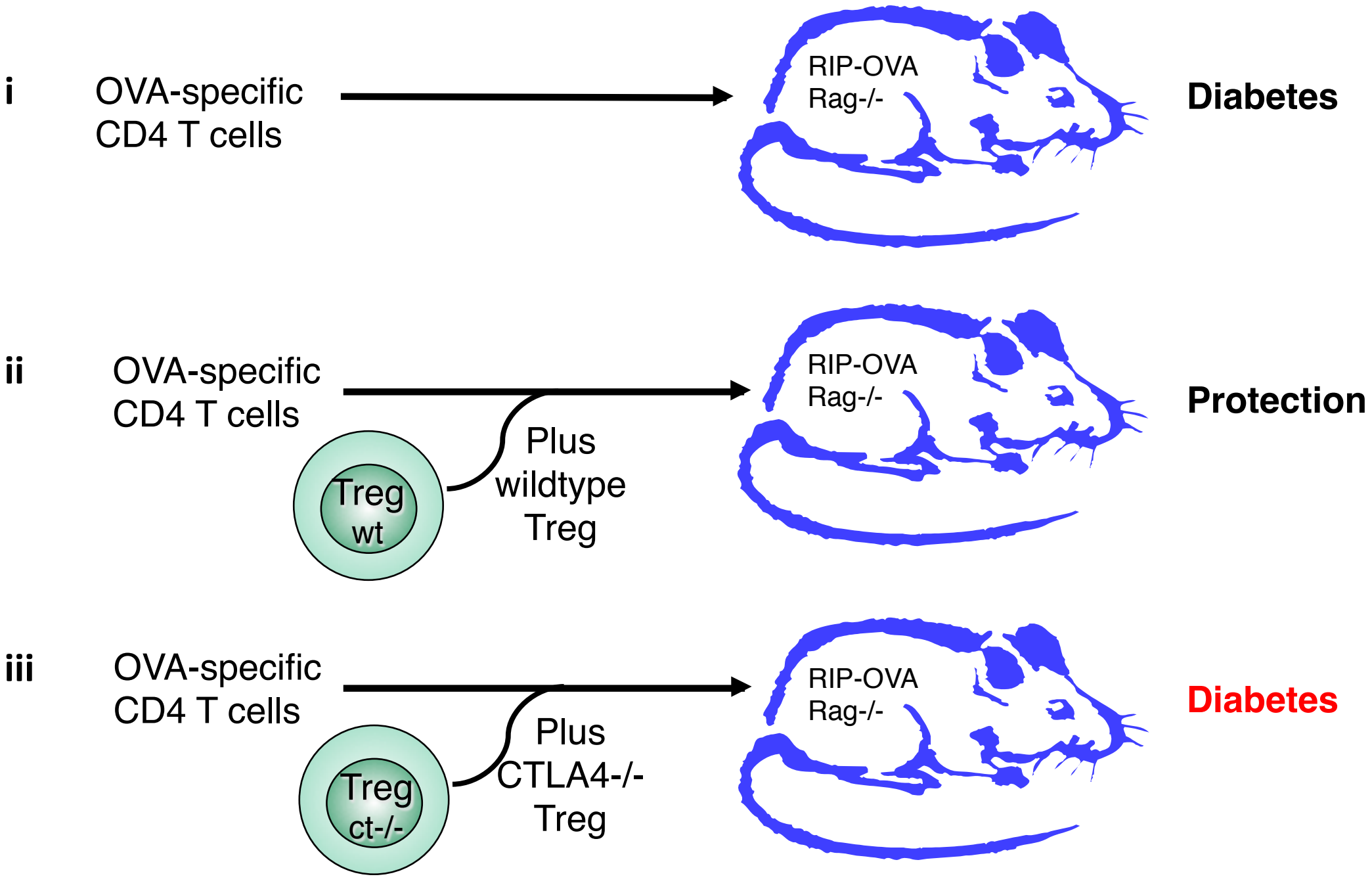

Figure 3. CTLA-4-/- antigen-specific Treg fail to control islet autoimmunity. i) Diabetes was induced by adoptively transferring OVA-specific T cells into rag2-/- mice expressing OVA in pancreatic beta-cells. ii) Co-transfer of OVA-specific Treg (isolated from DO11xrip-mOVA/rag-/- mice) protected from diabetes. iii) Co-transfer of CTLA4-deficient OVA-specific Treg (isolated from DO11xrip-mOVA/ctla4-/-/rag-/- mice) failed to confer disease protection (see ref 40 ). 

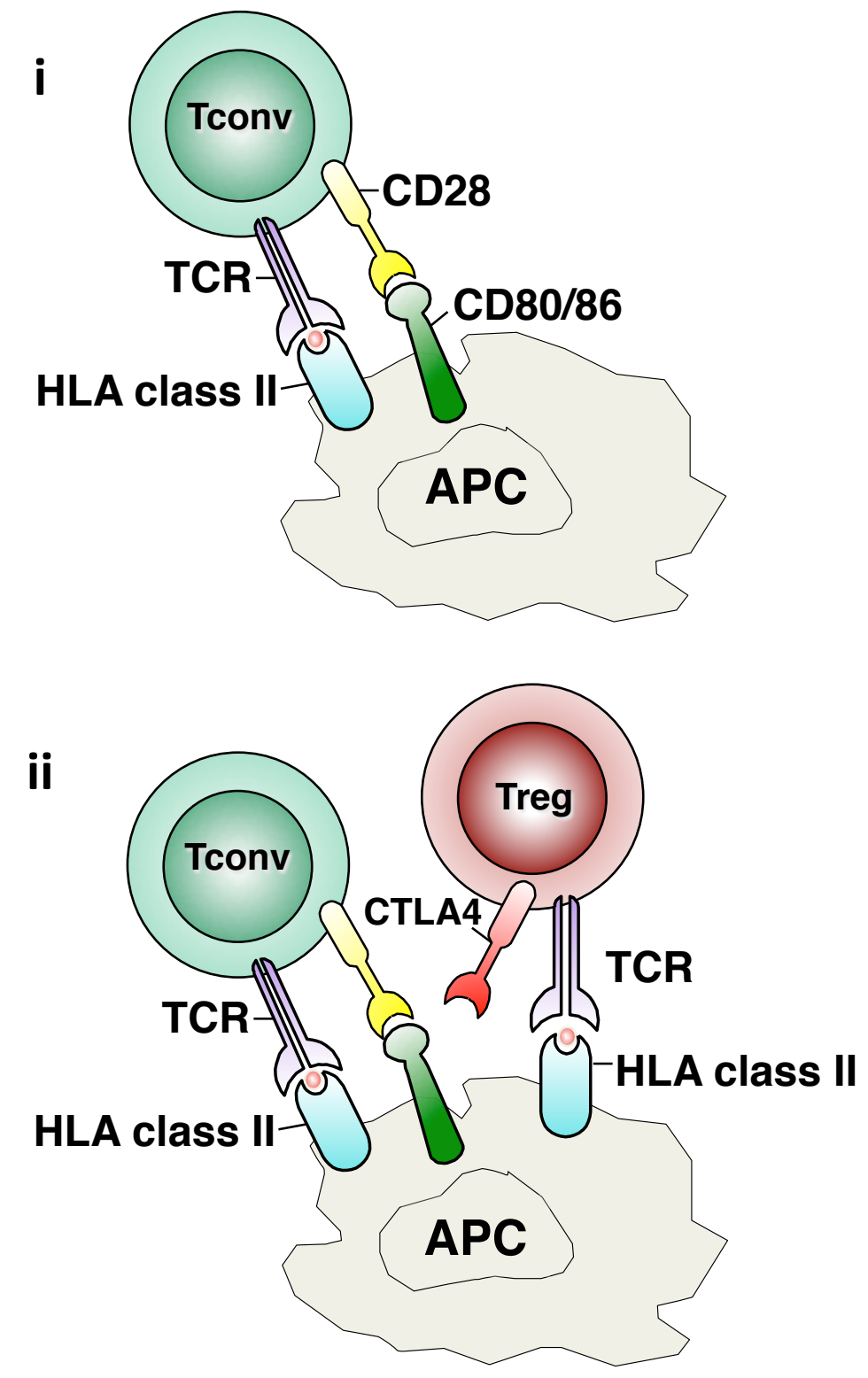

iii

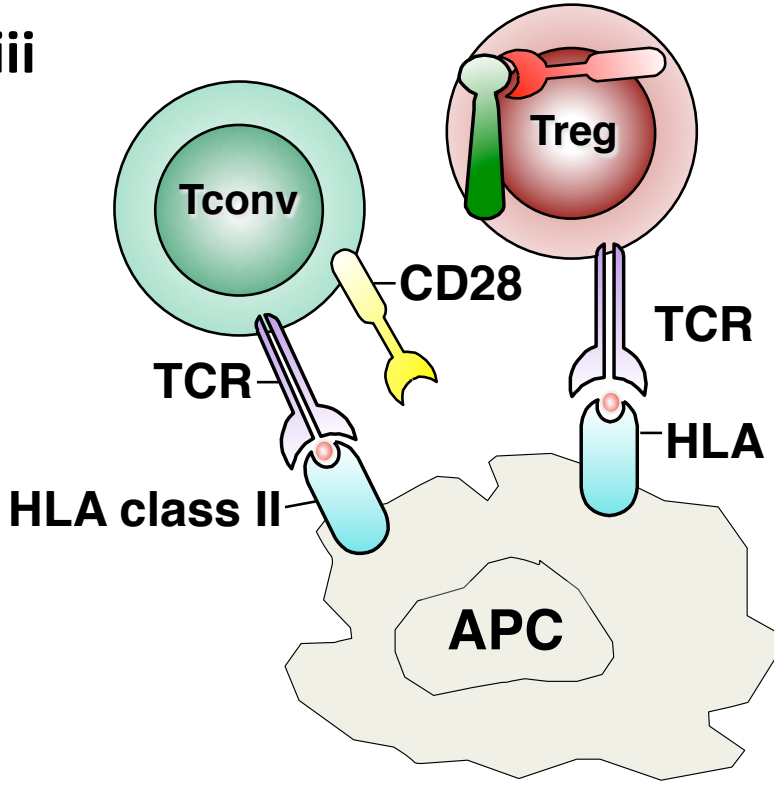

Figure 4. CTLA-4-mediated trans-endocytosis removes costimulatory ligands from APC. i) In the absence of Treg, APC can provide costimulatory ligands to activate Tconv. ii) Tregexpressed CTLA-4 binds to costimulatory ligands (CD80/86) and iii) removes them from the APC. This APC is now unable to provide costimulatory signals to activate Tconv. 